\title{
A Pipeline to Understand Emerging IIIness Via Social Media Data Analysis: Case Study on Breast Implant Illness
}

Vishal Dey ${ }^{1}$, BSc; Peter Krasniak ${ }^{2}$, MD; Minh Nguyen ${ }^{2}$, MD; Clara Lee ${ }^{2}$, MD; Xia Ning ${ }^{1,2,3}$, PhD

${ }_{1}^{1}$ Department of Computer Science and Engineering, The Ohio State University, Columbus, OH, United States

${ }^{2}$ Department of Biomedical Informatics, The Ohio State University, Columbus, OH, United States

${ }^{3}$ Translational Data Analytics Institute, The Ohio State University, Columbus, OH, United States

\section{Corresponding Author:}

Xia Ning, PhD

Department of Biomedical Informatics

The Ohio State University

1800 Cannon Drive

Columbus, OH, 43210

United States

Phone: 16143662287

Email: ning.104@osu.edu

\section{Abstract}

Background: A new illness can come to public attention through social media before it is medically defined, formally documented, or systematically studied. One example is a condition known as breast implant illness (BII), which has been extensively discussed on social media, although it is vaguely defined in the medical literature.

Objective: The objective of this study is to construct a data analysis pipeline to understand emerging illnesses using social media data and to apply the pipeline to understand the key attributes of BII.

Methods: We constructed a pipeline of social media data analysis using natural language processing and topic modeling. Mentions related to signs, symptoms, diseases, disorders, and medical procedures were extracted from social media data using the clinical Text Analysis and Knowledge Extraction System. We mapped the mentions to standard medical concepts and then summarized these mapped concepts as topics using latent Dirichlet allocation. Finally, we applied this pipeline to understand BII from several BII-dedicated social media sites.

Results: Our pipeline identified topics related to toxicity, cancer, and mental health issues that were highly associated with BII. Our pipeline also showed that cancers, autoimmune disorders, and mental health problems were emerging concerns associated with breast implants, based on social media discussions. Furthermore, the pipeline identified mentions such as rupture, infection, pain, and fatigue as common self-reported issues among the public, as well as concerns about toxicity from silicone implants.

Conclusions: Our study could inspire future studies on the suggested symptoms and factors of BII. Our study provides the first analysis and derived knowledge of BII from social media using natural language processing techniques and demonstrates the potential of using social media information to better understand similar emerging illnesses.

(JMIR Med Inform 2021;9(11):e29768) doi: 10.2196/29768

\section{KEYWORDS}

breast implant illness; social media; natural language processing; topic modeling

\section{Introduction}

\section{Background}

The ubiquity of social media has resulted in early descriptions of new and evolving diseases on social media platforms before they can be systematically studied [1-7], particularly during the era of the medical internet [8-14]. Social media users increasingly turn to platforms such as Twitter (Twitter Inc), Facebook (Facebook Inc), and YouTube (Google LLC) to share personal experiences, including diseases and illnesses they have experienced, or to seek support and resources, such as health and medical resources. Recent studies have shown the potential of social media in the detection of mental illness and depression [15-17] and in the early detection of food-borne illnesses [18-20] and other infectious diseases [2,21-24]. Furthermore, several 
studies have demonstrated social media as an effective tool to disseminate information regarding symptoms, personal well-being, and public health resources during multiple influenza outbreaks [25-28]. During the early stages of COVID-19, studies $[4,29,30]$ analyzed posts on Sina Weibo (Weibo Corporation) - a major Chinese microblogging site-to characterize patient symptoms and public concerns in multiple provinces of China. From the analysis of Weibo (Weibo Corporation) posts, Huang et al [30] concluded that most of the affected patients were older persons, with fever as the most common symptom. These studies demonstrate that public social media data can be leveraged to better understand emerging illnesses and to accommodate prompt responses.

One new illness we studied in this manuscript was breast implant illness (BII). Breast implants have gained popularity over the last 20 years [31]. During this period, more than 400,000 women have undergone breast augmentation or postmastectomy surgeries every year in the United States [32]. There was a 4\% increase in the number of breast augmentation procedures between 2017 and 2018, and a 6\% increase in breast implant removal procedures occurred over the same period [32]. Concerns about the safety of breast implants have also arisen [33-38] and persisted [39-45]. However, although a causal link between breast implants and systemic diseases has not been definitively shown, a phenomenon called breast implant illness, which attributes systemic symptoms to breast implants, has emerged [46]. Unlike other new medical illnesses, however, BII has been reported minimally in the medical literature, being primarily limited to social media [11,47-50]. For example, a recent analysis [49] demonstrated increasing public interest in BII based on Twitter and Google Trends data from February 2018 to February 2019. To summarize the key symptoms, diseases, and disorders defining BII, several cohort studies $[51,52]$ have analyzed patient-reported outcomes before and after breast explant surgeries. These studies showed some potential relationships between explant surgeries and the improvement of specific symptoms in the patient population. Unfortunately, these studies were not definitive because of their limited study design secondary to their lack of control groups, data collection bias, and lack of randomization. The lack of medical knowledge about BII makes it difficult to define the condition, and therefore, it is nearly impossible to conduct rigorous epidemiological or clinical studies. BII is just one disease process for which the lack of medical knowledge is apparent, but there are many other new illnesses for which this is the case. Any initial knowledge that is supported by sufficient social media data would be meaningful as a reference for formal studies in the future, and thus, the techniques to discover such knowledge are highly required.

\section{Objectives}

To identify and summarize the key attributes of a new illness, in this study, we constructed a data analysis pipeline for the social media data analysis of BII. The pipeline incorporated natural language processing (NLP) and topic modeling methods. Our primary objective is to derive novel knowledge about BII, a medical condition that has not yet been systematically studied and defined in the medical literature, by constructing a data analysis pipeline and applying the pipeline to social media data.
As medical knowledge and literature on BII have not been established and the related concepts are not well defined or accepted, using social media data to understand emerging issues could be a meaningful starting point. We applied this pipeline to better understand the symptoms and signs associated with BII. To the best of our knowledge, this study is the first to use social media data to derive the knowledge of BII from social media. This demonstrates the potential of using social media information to better understand the conditions that have primarily been reported on social media. It also establishes the effectiveness of our pipeline and its potential application to understand other new illnesses. In the following discussion, we have described our analysis pipeline in the context of BII. However, our pipeline is not specific to BII and is applicable to other illnesses as well.

\section{Methods}

\section{Data}

We collected and used data from select social media websites. These websites were selected because they were dedicated to BII discussions and information and were focused on user groups with interest in BII. Often, dedicated social media websites (eg, forums and Twitter pages) are available for a particular illness or disease. For example, some dedicated websites [53-55] contain the stories and experiences of patients fighting different cancers, some [56,57] contain posts and stories of users experiencing chronic pain and illness, and others [58-60] contain stories and experiences from COVID-19 survivors. The social media sources used in our study were as follows:

- $\quad$ BII [61]: This was a dedicated public website with articles on BII-related topics and offered resources related to implant and explant procedures, etc. This website also allowed individuals to post their experiences and concerns about breast implants and related health issues. We extracted individual posts from the website (up to May 10, 2019), and the resulting data set was referred to as BIIweb.

- Healing BII [62]: This website contained information on postimplant disorders, postexplant healing, breast implant safety, etc. The discussion board of this website had multiple posts and comments on symptoms, signs, etc, which are experienced by individuals with a breast implant or by those who have undergone an explant. The data set extracted from the discussion board of this website (up to May 10, 2019) was referred to as HealingBII.

- Instagram posts about BII [63]: This website contained a collection of publicly available Instagram posts that used breastimplantillness as a hashtag. We extracted the associated texts for each Instagram post with a timestamp between January 10, 2012, and September 4, 2019. The data set extracted from this site was referred to as IG-BII.

All the comments and posts from the 3 websites were included in the corresponding data sets. Table 1 presents a summary of the social media data collected. The BIIweb data set had only 187 posts (where each post on average has 129 words, SD 124) but these were larger (larger average length of posts in words) on average than those in the other 2 data sets. HealingBII was 
the second largest data set, with 1920 posts, each with 85 words on average $\left(l_{\text {avg }}\right)$ (SD 107). IG-BII was the largest data set, with

Table 1. Statistical summary of social media data analyzed.

\begin{tabular}{llllll}
\hline Data set & Posts $^{\mathrm{a}}(\mathrm{n}=31,094), \mathrm{n}(\%)$ & $1_{\max }^{\mathrm{b}}$ & $1_{\min }^{\mathrm{c}}$ & $1_{\text {avg }}^{\mathrm{d}}$, mean $(\mathrm{SD})$ & Words $^{\mathrm{e}}, \mathrm{n}(\%)$ \\
\hline BIIweb & $187(0.6)$ & 669 & 3 & $129(124)$ & $24,191(0.64)$ \\
HealingBII & $1920(6.17)$ & 1330 & 1 & $85(107)$ & $165,090(4.38)$ \\
IG-BII & $28,987(93.22)$ & 515 & 1 & $123(113)$ & $3,581,081(94.98)$ \\
\hline
\end{tabular}

${ }^{\text {a }}$ Posts: the number of posts and comments in the respective data sets.

${ }^{b} 1_{\max }$ : the minimum length of a post in words.

${ }^{c} 1_{\text {min }}$ : the maximum length of a post in words.

$\mathrm{d}_{l_{\text {avg }}}$ : the average length of posts in words.

e Words: the total number of words in the respective data sets.

\section{The Pipeline}

\section{Overview}

Figure 1 shows an overview of the pipeline. We extracted major topics of interest primarily related to symptoms, diseases, and medical procedures from our data sets through the following 3 steps. Each of the steps will be discussed in detail later. The first step involved data preprocessing. We removed all stop words, numeric characters, hyperlinks, hashtags, etc, and converted the remaining characters into lowercase. The second step was of mention extraction and concept mapping. We extracted mentions related to signs, symptoms, diseases, disorders, and medical procedures using the clinical Text Analysis and Knowledge Extraction System (cTAKES) [64]. The extracted mentions were further mapped to standard medical concepts represented by concept unique identifiers (CUIs) in the unified medical language system (UMLS) [65] ontology. The third step involved topic modeling. We summarized the

mapped concepts to topics using latent Dirichlet allocation (LDA) [66]. LDA is a probabilistic generative model for topic modeling. It represents each document as a mixture of latent topics, where each topic is modeled as a distribution over words. This modeling consisted of 3 stages: (1) mention replacement, (2) topic modeling using LDA, and (3) analysis and evaluation. In mention replacement, we replaced each extracted mention in the posts with its mapped CUIs and discarded all other words in the posts. We have discussed this step in more detail in the section Topic modeling. Then, in topic modeling using LDA, given the corpus of mapped CUIs, LDA generates document-topic and topics-CUI probability distributions. We have discussed this step in more detail in the section Topic modeling. Finally, during our analysis and evaluation, we further analyzed these distributions to derive a list of topics using the most representative mentions and summarized the extracted mentions for each data set. We have discussed this step in more detail in the section Results: LDA topics.

Figure 1. Pipeline for breast implant illness social media analysis. ASCII: American standard code for information interchange; CUI: concept unique identifier; LDA: latent Dirichlet allocation; cTAKES: clinical Text Analysis and Knowledge Extraction System.
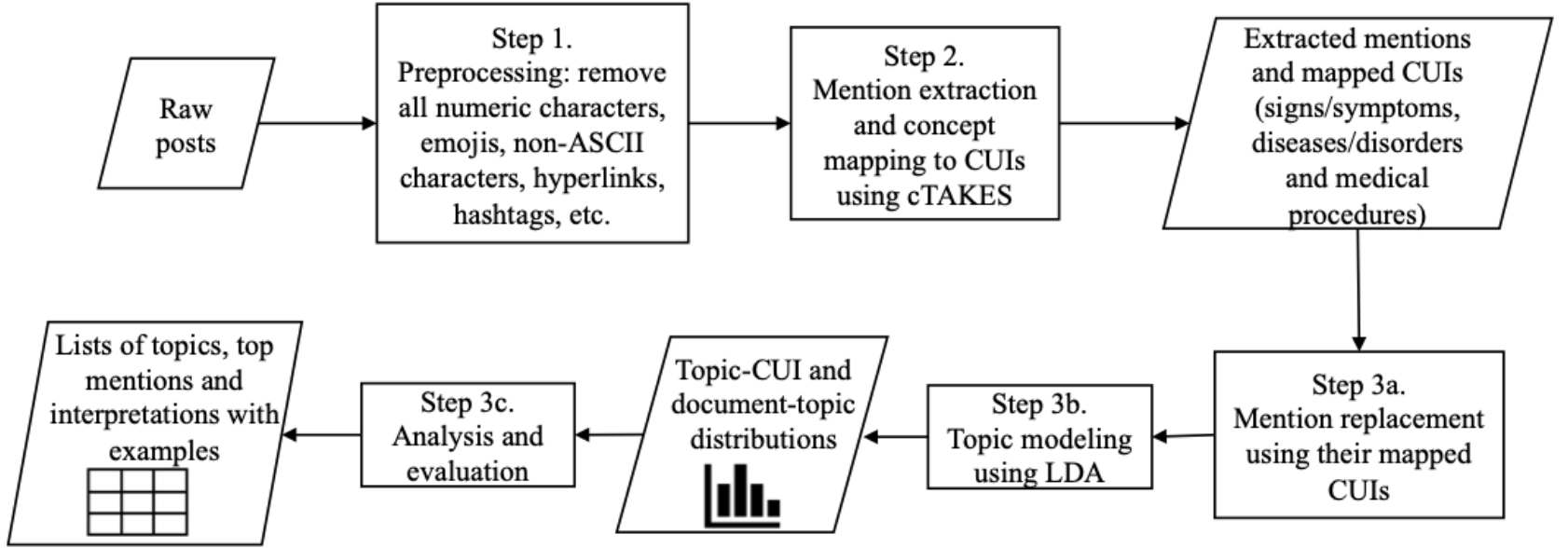

\section{Data Preprocessing}

We used the Natural Language Toolkit tokenizer [67] to tokenize the raw text for each data set. Out of the obtained tokens, we removed the stop-words (most frequently occurring, function words such as conjunctions, prepositions, determiners, etc) using the Natural Language Toolkit English stop-words list. As stop-words carried little or no information on our topics of interest in BII, they could be safely removed, as is typically done in NLP. We also removed all the numeric characters, emojis, non-American Standard Code for Information Interchange (ASCII) characters, hyperlinks, hashtags, and 
Instagram handles using regular expression matching and converted all the remaining tokens into lower cases to unify different cases for downstream processing.

\section{Mention Extraction and Concept Mapping}

Mention extraction refers to the extraction of words or phrases that convey a medical concept. We used the cTAKES tool for mention extraction. The cTAKES tool is an open-source NLP tool for clinical information extraction from unstructured clinical texts. cTAKES extracts mentions (ie, words or phrases that convey a medical concept) from posts and maps these mentions to standard medical concepts. In doing so, it also categorizes each extracted mention into one of 5 cTAKES categories: sign, symptom, disease, disorder, medication, procedure, and anatomy; that is, while cTAKES extracts mentions, it also automatically classifies the mentions into one of the 5 categories. For example, in the sentence "Over the years, my tinnitus has become worse to almost debilitating levels," cTAKES extracts tinnitus as a mention of sign and symptom category. Below, we discuss how to configure the cTAKES in detail.

We used the fast-dictionary-lookup annotator in cTAKES to extract mentions from the processed data. This annotator identifies and extracts mentions in texts and normalizes them into CUIs in the UMLS standard medical ontology. This normalization of extracted mentions into CUIs is referred to as concept mapping. Each CUI in the UMLS ontology uniquely identifies a medical concept. Hence, we represented extracted mentions using the standard medical concepts of CUIs that cTAKES maps the mentions to. We configured the annotator to use an exact string match and to use the all-term-persistence property. Thus, the annotator could retain all terms, irrespective of the semantic properties of each term. For example, for the phrase back pain, the annotator would annotate the generic term pain as well as the precise term back pain. We chose to use the all-term-persistence property to retain maximum information with respect to precise and generic medical concepts. Finally, the annotator stored the generated annotations in XML Metadata Interchange (XMI) files.

To obtain the annotations in a human-readable format from the XMI files, we performed the following steps (Figure 2). We used a custom interpreter to process the XMI files produced by cTAKES and to obtain mappings between mentions and CUIs from cTAKES. We first searched for UmlsConcept XML identifiers in the XMI files, where each UmlsConcept XML identifier is generally grouped under the FSArray, and each FSArray is associated with a single ontology concept and the category of the concept. Each concept is assigned one category out of 5 cTAKES categories: sign, symptom, disease, disorder, medication, procedure, and anatomy. Each ontology concept is further associated with a UMLS CUI and an ontologyConceptArr identifier. It must be noted that a mention can be mapped to multiple CUIs. For example, the mention allergic reaction is categorized as sign and symptom but mapped to 2 different CUIs: C1527304 and C0020517. Then, we extracted the ontology concepts that describe any of these categories: diseases, disorders, signs, symptoms, and medical procedures. Finally, we used the begin and end markers associated with each ontologyConceptArr identifier to obtain the position of the annotated mention in the input post. In this work, we were only interested in the first 3 categories (ie, sign, symptom, disease, disorder, and procedure) to understand BII-related issues. Hence, we only used the mentions categorized into either of these 3 categories.

Figure 2. Pipeline for obtaining annotations out of Clinical text analysis and knowledge extraction system. cTAKES: clinical Text Analysis and Knowledge Extraction System; CUI: concept unique identifier; UMLS: unified medical language system.

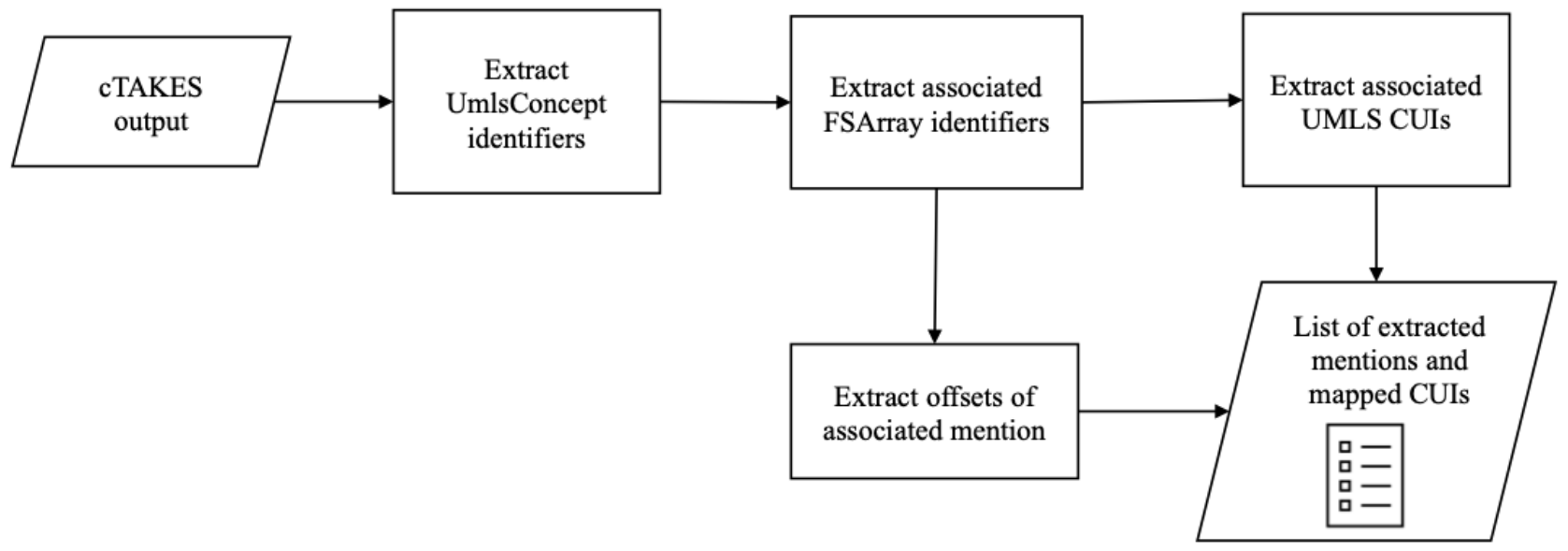

\section{Topic Modeling}

To conduct topic modeling, we processed the posts as follows: we substituted each mention in the posts with its mapped CUIs and discarded all other words in the posts, which were considered as nonmedical concepts by cTAKES or were not among the 3 categories of interest. If a mention was mapped to multiple CUIs, we replaced it with multiple CUIs. If multiple mentions were mapped to the same CUI, we replaced all such mentions with the CUI. In this way, each post was represented as a bag-of-CUI, instead of a collection of mentions, as the input to the topic modeling and our vocabulary consisted of CUIs. Upon topic modeling, we interpreted the topic-CUI distribution to derive the topics.

We used LDA [66] to learn the topic distributions of each post and the CUI distributions of each topic. LDA is a generative probabilistic model for modeling topics within a document corpus. LDA models each document in the corpus as a mixture 
of latent topics, where each topic is modeled as a distribution over words in all documents. LDA derives the optimal distributions by maximizing the likelihood of observing the corpus, following perspective distributions. A brief description of LDA is provided in Multimedia Appendix 1 [66]. In our experiments, a bag-of-CUIs generated as described above was used as a document in LDA, and the CUIs were words in the document. We used the lda-c software [68], which is a very efficient implementation of the LDA method, to conduct topic modeling.

When LDA is used in topic modeling for general documents (eg, news, scientific literature), words and their frequencies in the documents are used. However, in our analysis, we aimed to understand the medical concepts related to BII from social media texts. Different words may indicate the same medical concepts. For example, joint aches, painful joints, arthralgia, and aching joints all indicate joint pain and are associated with a single medical concept represented by a single CUI. Therefore, instead of using words, we used medical concepts, represented by CUIs, in our LDA analysis. Because multiple words indicating the same medical concept can be mapped to the same CUI, using
CUIs can also aggregate and strengthen the information from multiple words, compared with using words, which may be sparse and thus not easy to learn topics from.

\section{Results}

\section{cTAKES Annotations}

Table 2 presents the summary statistics for the annotated mentions and their CUIs mapped by cTAKES. In BIIweb, cTAKES extracted 2186 mentions and mapped them to 475 unique CUIs. In HealingBII, cTAKES extracted 11,080 mentions and mapped them to 1177 unique CUIs. In the largest data set IG-BII, cTAKES extracted 5530 unique mentions and mapped them to 2871 unique CUIs. Note that the same mention can be mapped to multiple CUIs and can have multiple categories (each CUI has only one category). For example, the mention flashes is mapped to 2 different CUIs and then 2 different categories: diseases and medical procedures. Table 2 presents the statistics for each category of extracted mentions. For each data set, most of the extracted mentions were categorized as signs and symptoms by cTAKES.

Table 2. Statistical summary of annotations of the clinical Text Analysis and Knowledge Extraction System.

\begin{tabular}{lllllllllll}
\hline Data set & cwords $^{\mathrm{a}}$ & annots $^{\mathrm{b}}$ & maps $^{\mathrm{c}}$ & $\mathrm{M}^{\mathrm{d}}$ & $\mathrm{C}^{\mathrm{e}}$ & $\mathrm{M} / \mathrm{C}^{\mathrm{f}}$ & ${\mathrm{C} / \mathrm{M}^{\mathrm{g}}}^{\mathrm{S}^{\mathrm{h}}}$ & $\mathrm{D}^{\mathrm{i}}$ & $\mathrm{P}^{\mathrm{j}}$ \\
\hline BIIweb & 24,034 & 2186 & 661 & 640 & 475 & 1.39 & 1.03 & 385 & 149 & 106 \\
HealingBII & 163,352 & 11,080 & 1740 & 1685 & 1177 & 1.48 & 1.03 & 891 & 503 & 292 \\
IG-BII & $3,116,966$ & 185,339 & 5694 & 5530 & 2871 & 1.98 & 1.03 & 3049 & 1549 & 932 \\
\hline
\end{tabular}

a cwords: the total number of words recognized by the clinical Text Analysis and Knowledge Extraction System.

bannots: the total number of extracted mentions belonging to the 3 semantic types (ie, signs, symptoms, diseases, disorders, and medical procedures).

${ }^{c}$ maps: the number of unique mention-concept unique identifier mappings.

${ }^{\mathrm{d}} \mathrm{M}$ : the number of unique extracted mentions.

${ }^{\mathrm{e}} \mathrm{C}$ : the number of unique mapped concept unique identifiers.

${ }^{\mathrm{f}} \mathrm{M} / \mathrm{C}$ : the average number of extracted mentions mapped to a given concept unique identifier.

${ }^{\mathrm{g}} \mathrm{C} / \mathrm{M}$ : the average number of concept unique identifiers mapped to an extracted mention.

${ }^{\mathrm{h}_{\mathrm{S}}} \mathrm{S}$ the number of unique extracted mentions mapped to the signs and symptoms category.

${ }^{\mathrm{i}} \mathrm{D}$ : the number of unique extracted mentions that are mapped to the diseases and disorders category.

${ }^{\mathrm{j}} \mathrm{P}$ : the number of unique extracted mentions mapped to the medical procedures category.

To determine if cTAKES can sufficiently extract relevant mentions, we performed a manual annotation and compared the 2 lists of extracted mentions: one from using cTAKES and the other from using manual annotation. We randomly sampled 50 posts from each of the 3 data sets and manually annotated these posts. Upon manual annotation, we extracted mentions (words or phrases) that conveyed the concerns and experiences of social media users involving BII-related symptoms, diseases, and medical procedures. For a random sample of 50 posts $\left(l_{\mathrm{avg}}=134.18\right)$ from BIIweb, we obtained a total of 575 mentions from using manual annotation, and 637 mentions using cTAKES; there were 479 common mentions. Each mention was associated with a post identifier and a character offset. A mention was considered to belong to both lists if it occurred in both lists with the same post identifier and character offset. We found that $83.3 \%$ (479/575) of manually annotated mentions were covered by cTAKES. This high coverage demonstrates that cTAKES can capture most of the relevant medical concepts.
In contrast, $75.2 \%(479 / 637)$ of the annotated mentions by cTAKES were covered by manual annotation. This further demonstrates that most of the annotated mentions of cTAKES can be confirmed by manual annotation. Similarly, for a random sample of 50 posts $\left(1_{\mathrm{avg}}=80.02\right)$ from HealingBII, $69.5 \%$ $(194 / 279)$ of manually annotated mentions were covered by cTAKES; $70.3 \%$ (194/276) of mentions annotated by cTAKES were confirmed by manual annotation. For a random sample of 50 posts $\left(\mathrm{l}_{\mathrm{avg}}=121.00\right)$ from IG-BII, the corresponding values were $75.2 \%(182 / 242)$ and $64.3 \%(182 / 283)$, respectively. According to the high overlap in the results between manual annotation and cTAKES across multiple data sets used in our study, it is reasonable to assume that cTAKES is a decent surrogate of manual annotation for BII study through social media data. 


\section{LDA Topics}

To identify the best topic models, we used a grid search to identify the best parameter values for the Dirichlet prior $\alpha \in$ $\{0.01,0.05,0.1,0.5,1,1.5,2,5,10,15,20,25\}$ and the number of topics $\mathrm{K} \in\{3,4,5,10,15,20\}$. To evaluate topic models, we analyzed each LDA topic modeling result for every combination of $\alpha$ and $\mathrm{K}$ values corresponding to low perplexity scores $[66,69,70]$.

For each topic modeling result, we analyzed the document-topic and topic-CUI probability distributions to derive topics and their respective top 10 representative mentions. The top 10 representative mentions for a given topic were the most frequent mentions, corresponding to the top 10 CUIs, with the highest probabilities of belonging to the topic. Multiple mentions could be mapped to a given CUI (Table 2). We only presented the most frequent mention because all mentions mapped to the same CUI had similar semantics. We further evaluated the quality of topic modeling based on how well the derived topics summarized the most representative mentions. We analyzed each LDA topic modeling result for every combination of $\alpha$ and $\mathrm{K}$ and chose the one where the derived topics were distinct and best summarized the most representative mentions. Finally, we identified distinct and meaningful topics using (1) $\mathrm{K}=4$ and $\alpha=10$ for BIIweb, (2) $K=5$ and $\alpha=10$ for HealingBII, and (3) $\mathrm{K}=5$ and $\alpha=1.5$ for IG-BII. We observed that with higher $\mathrm{K}$ values, the most representative mentions were similar across the topics. Hence, the derived topics were not distinct and were difficult to interpret.

Tables 3-5 present the top 10 representative mentions, the frequencies of CUIs corresponding to the mentions (in \%), and the interpretations of the topics indicated by the mentions (eg, common signs and symptoms). Note that the frequencies of CUIs are among all the posts, not only in those posts with the highest probability belonging to a certain topic. We presented these frequencies because each post had a certain probability of belonging to a certain topic, and thus frequencies among all posts would better represent the topic information across all the posts. These tables also present examples of posts that have a high probability of belonging to the respective topic. In the examples, the mentions that had high probabilities of belonging to the corresponding topics are italicized. Note that we used CUIs in LDA to derive the topic and word distributions (as discussed in the section Methods-Topic modeling), but we have presented the most frequent mentions (with clear semantics) that were mapped to the respective CUIs (which are identifiers without semantics) in these tables. The mentions in these tables were sorted based on the probabilities of their corresponding CUIs belonging to the respective topics. Please note that these probabilities have not been presented in the tables (they are not the frequencies presented in the tables). Therefore, each topic was represented by its most representative mentions, and thus, summarized such mentions. For example, we interpreted a topic as pain and other signs if there were a significant number of mentions related to pain, such as neck pain, chest pain, and headache. Please note that the topics have not been sorted, and the first columns in Tables 3 to 5 are nominal identifiers. Below, we have discussed the topics derived from LDA for BIIweb and HealingBII data sets from the original posts. Note that 2 topics can still share the same representative mention with different probabilities in the LDA.

Table 3. Derived topics in BIIweb.

\begin{tabular}{|c|c|c|}
\hline Topic & Top 10 mentions & Interpretation \\
\hline 1 & $\begin{array}{l}\text { - Testing (2.34); illness }(4.46) \text {; problem }(2.82) \text {; work }(1.17) \text {; swollen }(0.78) \text {; drains }(0.61) \text {; feel common } \\
\text { (2.51); fatigue (1.82); exhausted }(0.39) \text {; sensitivity }(0.95) \\
\text { Example: "I had silicone implants done } 5 \text { years ago, three years ago after going to the doctor with } \\
\text { extreme fatigue (I was sleeping } 14-16 \text { hours a day and was still exhausted)" }\end{array}$ & Common signs and symptoms \\
\hline
\end{tabular}

- $\quad$ Breast implant (6.80); removal (1.30); cancer (0.95); autoimmune (0.95); infection (0.87); scleroderma (0.39); pain (3.68); diagnosis (0.30); alcl (0.30); breast cancer $(0.30)$

- $\quad$ Example: "I had stage 4 breast cancer and had chemo and radiation. I tried to have my breast implants removed due to pain...Then I had an acute infection occur a month and a half after they put the new implants in and they were forced to perform an emergency removal of the newer implants. I have had all the symptoms of breast implant illness-even after their removal."

- $\quad$ Breast implant (6.80); illness (4.46); toxicity (1.17); foreign body (0.87); heal (0.78); support (0.65); Toxicity rupture (0.52); cancer (0.95); awareness (0.35); inflammation (0.56)

- Example: “...I never had a problem until 2006 at which time I thought something had happened however, my surgeon said I must have just pulled a muscle and that the implants seemed fine. Now that surgeon is old and the shop is closed up. I have been suffering for the past 13 years with arthritis, fatigue, brain fog, inflammation, hormone imbalances, and adrenal fatigue..."

- $\quad$ Pain (3.68); feel (2.51); fatigue (1.82); back pain (0.87); illness (4.46); joint pain (0.56); worse (0.65); anxiety (0.52); ear ringing (0.39); headache (0.39)

Diseases or disorders 
Table 4. Derived topics in HealingBII.

\begin{tabular}{|c|c|c|}
\hline Topic & Top 10 mentions & Interpretation \\
\hline 1 & $\begin{array}{l}\text { - } \quad \text { Rupture (1.34); supported }(0.87) \text {; read }(1.17) \text {; suffering }(0.87) \text {; happy }(0.6) \text {; mastectomy }(0.46) \text {; work } \\
\text { - } \quad \text { Example: "Double mastectomy }{ }^{\mathrm{a}} \text { in } 2015 \text {. Reconstruction process with expanders then permanent } \\
1000 \mathrm{ml} \text { saline implants in early } 2016 . \text { After that was } 9 \text { procedures, a hysterectomy and now MANY } \\
\text { health problems." }\end{array}$ & Surgeries and procedures \\
\hline 2 & $\begin{array}{l}\text { - Pain (3.91); joint pain (0.79); fatigued (0.96); ailment (4.70); removal (0.84); hair loss (0.52); headache } \\
\text { - } 0.47) \text {; muscle ache (0.34); rash (0.39); infection (0.84) } \\
\text { Example: "In addition to the neuromuscular spasms and pain, I've suffered with incapacitating } \\
\text { chronic fatigue, BRAIN FOG and confusion (yes, even while driving), loss of vision and hearing, } \\
\text { vertigo, mysterious skin rashes, hair loss, migraines..." }\end{array}$ & Pain and other signs \\
\hline 3 & $\begin{array}{l}\text { - Problem (2.64); cancer }(0.90) \text {; autoimmune }(0.57) \text {; breast cancer }(0.38) \text {; scars }(0.35) \text {; treatment }(0.43) \text {; } \\
\text { diagnose }(0.29) \text {; autoimmune disorder }(0.27) \text {; lupus }(0.29) \text {; arthritis }(0.26) \\
\text { Example: "I had capsules form on both breasts from about } 2010 . \text { I got sick with BII symptoms from } \\
2005 \text { with lots of infections required intravenous and oral antibiotics. My environmental and drug } \\
\text { allergies got worse, onset of arthritis, skin rashes, autoimmune symptoms, started growing low grade } \\
\text { cancers..." }\end{array}$ & Cancer and other disorders \\
\hline 4 & $\begin{array}{l}\text { - Breast implant (3.85); ailment (4.70); toxicity (3.05); healing (1.56); capsulectomy (0.64); infection } \\
\text { - } 0.84) \text {; inflammation }(0.39) \text {; detoxification }(0.32) \text {; foreign object }(0.25) \text {; bleed }(0.23) \\
\text { Example: "Some women with silicone toxicity have bruising and bleeding problems. If I was you, I } \\
\text { would try and have the lymph node localized and checked for silicone and removed if it is contami- } \\
\text { nated beyond detoxing much like a silicone granuloma is removed." }\end{array}$ & Toxicity \\
\hline 5 & $\begin{array}{l}\text { - Emotion (3.70); think (2.26); feel (0.84); normal (0.65); anxiety }(0.50) \text {; ill }(0.61) \text {; sensation }(0.33) \text {; } \\
\text { tired (0.28); sores }(0.27) \text {; depression }(0.33) \\
\text { - Example: "Even more heartbreaking and discouraging, has been the emotional pain of not being able } \\
\text { to freely play with her on the floor due to hip and knee pain, along with leg and foot spasms...but I } \\
\text { struggle with many feelings of failure as a wife and mother due to physical limitations." }\end{array}$ & Mental health \\
\hline
\end{tabular}

${ }^{a}$ Italic text indicates the mentions in the examples that had high probability of belonging to the corresponding topics. 
Table 5. Derived topics in IG-BII.

\begin{tabular}{|c|c|c|}
\hline Topic & Top 10 mentions & Interpretation \\
\hline 1 & $\begin{array}{l}\text { - Heal (1.46); working ( } 0.90) \text {; weighted (1.05); able (0.99); rest }(0.37) \text {; stress }(0.29) \text {; exercise }(0.28) \text {; } \\
\text { therapeutic (0.35); sleep (0.36); run ( } 0.23) \\
\text { Example: "It's been } 14 \text { months since my explant. The journey to healing }{ }^{\text {a }} \text { hasn't been an easy one } \\
\text { due to setbacks and relapses but better than daily anaphylaxis from getting cold, food, smells, crying, } \\
\text { exercise and stress, then add angina attacks from anaphylaxis." }\end{array}$ & Physical health \\
\hline
\end{tabular}

- Malignancy (1.10); removal (0.96); scar (0.75); capsulectomy (0.68); rupture (0.43); ciactrice (0.43); alcl (0.41); augmentation (0.37); lymphoma (0.35); removal of implants (0.29)

Cancer and medical procedures

- Example: "The ugly side of breast implants. It's not a matter of IF you will get sick...it's WHEN. implants leak toxic heavy metals without rupture It's called a gel bleed. Women with implants are 3 times more likely to develop brain, lung and lymphatic cancer than women with implants." (0.35); tired (0.38); emotional (0.27); sensation (0.33)

- $\quad$ Example: "I was scared of looking incomplete. After much deep, inner work on myself, I realized that my worth wasn't dependent on what I looked like or how big my chest was. I realized that true happiness would come from $100 \%$ acceptance of what and who I was"

- $\quad$ Breast implant (7.21); ailment (5.67); toxicity (1.67); aware (0.96); felt worse (0.36); test (0.64); foreign body (0.45); alone (0.33); suffering $(0.21)$; complication $(0.20)$

- Example: "...We get toxic from the chemical makeup of the silicone, the toxic chemicals that are released when the shell degrades, sick from rupture and sometimes mold."

- Pain (2.52); inflammatory reaction (0.89); fatigue (0.83); anxiousness (0.72); allergy (0.43); depression (0.37); joint pain (0.33); autoimmune disorder (0.32); swell (0.43); infection $(0.31)$

- Example: "For three years, doctors have been unable to diagnose or explain upper body weakness, hand pain, and general inflammation. I have suffered from periods of high inflammation, debilitating fatigue, migraines, inability to lose weight, insomnia, low libido, body and joint pain, hair loss, dry skin, dry eyes, brain fog, etc."

Mental health

(1) 
Table 6. Derived topics in the unified data set.

\begin{tabular}{|c|c|c|}
\hline Topic & Top 10 mentions & Interpretation \\
\hline 1 & $\begin{array}{l}\text { - Working (1.45); ate }(0.92) \text {; weight }(0.79) \text {; runs }(0.40) \text {; thinking }(2.68) \text {; exercise }(0.25) \text {; talk }(0.50) \text {; } \\
\text { walking }(0.35) \text {; nutrition }(0.15) \text {; move }(0.28) \text {; } \\
\text { Example: “...I'm now healthier than I have been in the last } 7 \text { years of my life!...I explanted in Feb } \\
\text { of } 2018 \text {, a few months after explant, I gained my weight }{ }^{\mathrm{a}} \text { back and found a love for true self care } \\
\text { and working out.” }\end{array}$ & Physical health \\
\hline 2 & $\begin{array}{l}\text { - Illnesses (4.45); cancer ( } 0.87) \text {; ruptures }(0.77) \text {; removal }(0.76) \text {; awareness }(0.73) \text {; suffers }(0.83) \text {; } \\
\text { capsulectomy (0.54); autoimmune }(0.52) \text {; breast augmentation }(0.30) \text {; augmentation }(0.28) \text {; } \\
\text { Example: "I was diagnosed with breast cancer at the young age of } 30 \text { and ended up with a double } \\
\text { mastectomy as part of that process...now } 10 \text { years later I have just } 15 \text { weeks ago had my implants } \\
\text { removed. They had ruptured, were toxic and giving me health issues" }\end{array}$ & $\begin{array}{l}\text { Cancer and medical proce- } \\
\text { dures }\end{array}$ \\
\hline 3 & $\begin{array}{l}\text { - Feel (5.94); loved (2.97); thinking (2.68); happier (1.64); feelings (1.47); afraid (0.66); confidence } \\
\text { (0.27); support ( } 0.79) \text {; able (0.77); alive (0.17); } \\
\text { Example: "When I found out I was sick and I had to tear apart my body to get better I never thought } \\
\text { I'd be happy with myself again. I am } 4 \text { weeks post op and feeling more happy and healthy than ever. } \\
\text { I was worried I'd never be loved again." }\end{array}$ & Mental health \\
\hline 4 & $\begin{array}{l}\text { - Heal (2.26); scars }(0.58) \text {; scarred }(0.33) \text {; drain }(0.26) \text {; toxic }(1.97) \text {; sights }(1.25) \text {; inflammation }(0.68) \text {; } \\
\text { bulge }(0.36) \text {; tenderness }(0.20) \text {; red }(0.15) \text {; damage }(0.16) \text {; } \\
\text { Example: "I was so worried about how red and raised up my scars were...then they got really inflamed, } \\
\text { sore and raised up around } 3 \text { weeks and i was really stressed over it. then overnight the inflammation } \\
\text { and redness went down..." }\end{array}$ & $\begin{array}{l}\text { Common signs, symptoms, } \\
\text { and toxicity }\end{array}$ \\
\hline 5 & $\begin{array}{l}\text { - Pain (2.09); tired all the time (0.69); anxiety }(0.57) \text {; joint pain }(0.46) \text {; alopecia }(0.39) \text {; weight gain } \\
\text { (0.37); allergies }(0.35) \text {; depression }(0.29) \text {; pain back }(0.23) \text {; headache }(0.22) \\
\text { Example: "Before I had the explant, I had many unexplained symptoms (brain fog, joint pain, back } \\
\text { and neck pain, tired all the time, psoriasis, afib, just to mention a few) since I awoke from surgery I } \\
\text { have had absolutely no neck, back, or joint pain." }\end{array}$ & Common disorders \\
\hline
\end{tabular}

${ }^{\mathrm{a}}$ Italic text indicates the mentions in the examples that had high probability of belonging to the corresponding topics.

Table 7 presents the percentage of posts per topic, where a post $d$ is considered to belong to a topic $\mathrm{z}$ if among all topics that $d$ has, $\mathrm{z}$ has the highest probability. Although the distributions are not completely consistent across data sets, toxicity remained a notable topic among all data sets. This indicates that these were common issues that were significantly associated with BII. In addition, pain, cancer, mental health, and other disorders were also associated with breast implants. 
Table 7. Distribution of posts among the topics.

\begin{tabular}{|c|c|}
\hline Data set and topics & Posts, n (\%) \\
\hline \multicolumn{2}{|l|}{ BIIweb } \\
\hline Common signs and symptoms & $62(33.2)$ \\
\hline Diseases or disorders & $28(15)$ \\
\hline Toxicity & $50(26.7)$ \\
\hline Pain and stress-related disorders & $47(25.1)$ \\
\hline \multicolumn{2}{|l|}{ HealingBII } \\
\hline Surgeries and procedures & $713(37.1)$ \\
\hline Pain and other signs & $221(11.5)$ \\
\hline Cancer and other disorders & $221(11.5)$ \\
\hline Toxicity & $505(26.3)$ \\
\hline Mental health & $260(13.6)$ \\
\hline \multicolumn{2}{|l|}{ IG-BII } \\
\hline Physical health & $11,299(39)$ \\
\hline Cancer and medical procedures & $3890(13.4)$ \\
\hline Mental health & $4879(16.8)$ \\
\hline Toxicity & $5415(18.7)$ \\
\hline Common disorders & $3504(12.1)$ \\
\hline \multicolumn{2}{|l|}{ Unified } \\
\hline Physical health & $4760(15.3)$ \\
\hline Cancer and medical procedures & $10,637(34.2)$ \\
\hline Mental health & 7954 (25.6) \\
\hline Common signs, symptoms, and toxicity & $4030(13)$ \\
\hline Common disorders & $3713(11.9)$ \\
\hline
\end{tabular}

\section{Discussion}

\section{Principal Findings}

To understand the signs, symptoms, and diseases or disorders associated with BII, a condition reported primarily on social media rather than in medical reports, we collected social media posts and analyzed them using NLP and topic modeling. We extracted mentions related to signs, symptoms, diseases, disorders, and medical procedures using cTAKES, mapped them to standard medical concepts, and summarized the mapped concepts to topics using LDA. We found that mentions such as rupture, infection, inflammation, pain, and fatigue were common self-reported issues. We also found that mental health-related concerns such as stress, anxiety, and depression, as well as diseases such as cancers and autoimmune disorders, were common concerns. The cTAKES was able to extract medication and anatomy information as well, but they were not used in our LDA analysis, given that the objective of our study was not to study the medications used or the anatomy related to BII.

In our method, we relied on cTAKES and the rich UMLS dictionary to extract all relevant mentions, including their lexical variants (synonyms, abbreviations, paraphrases). To determine if cTAKES could sufficiently extract relevant mentions, we performed a manual annotation to extract all the relevant mentions and compared them with the extracted mentions from cTAKES. We found that cTAKES could sufficiently capture relevant medical concepts and was comparable with manual annotation. It is worth noting that we did not evaluate the performance of our mention extraction module on all the posts of each data set, which is typically performed using precision and recall metrics when there are ground-truth labels associated with each mention. However, in order to have such labels, careful manual annotations based on domain knowledge of BII are required. Unfortunately, such domain knowledge on complications, symptoms, and other issues associated with or caused by BII were not fully available. Our goal in this study is to provide useful information from social media data that could complement our current knowledge. Therefore, in this preliminary study, we used all annotated mentions, assuming that cTAKES enabled high-quality annotations.

\section{Strengths and Limitations}

We acknowledge that cTAKES might not have been able to extract all relevant mentions from our social media data sets. This is because cTAKES was originally designed for extraction of medical entities from clinical notes, which have very different wording and writing styles compared with social media data. As social media data comprise informal phrases, short ambiguous texts, emoticons, and a wide range of lexical variants 
corresponding to a single concept, cTAKES might not work flawlessly on social media data, although we observed reasonable output from cTAKES. We also observed that cTAKES often associated a single mention with multiple CUIs belonging to the same category. We think this was because of the presence of multiple mappings for a given mention in the UMLS metathesaurus. Regardless, the extracted mentions and the mapping of mentions to UMLS CUIs, as generated by cTAKES, were used for topic modeling without any manual verification or evaluation. In the future, we will develop a detailed guideline to further evaluate the extracted mentions before using them in topic modeling.

Our study had some limitations. First, LDA is an unsupervised learning technique in which the number of topics $(\mathrm{K})$ is assumed to be known a priori. However, it is difficult to accurately estimate $\mathrm{K}$ for a given data set. In our study, we used a grid search to obtain different $\mathrm{K}$ values. Even without full domain knowledge, it remains nontrivial to evaluate the LDA results for each $\mathrm{K}$ value. In our study, we selected the topics based on $\alpha$ and $\mathrm{K}$ values. We did not use perplexity $[66,69,70]$, a widely used metric in topic modeling, to select the topics, because as studied in the literature (eg, Chang et al [71]), perplexity often does not correlate well with topic interpretability; in our case, the lowest perplexity did not always enable intuitive or meaningful topics. In the future, we will develop more rigorous ways to select the number of topics and evaluate the topic modeling results. In this study, we did not conduct a sentiment analysis of the posts to understand the positive or negative opinions expressed in the posts. We plan to include this process before topic modeling to generate a cleaner data set for topic modeling.

It is worth noting that social media data could be of variable quality (eg, misspelling, misconception, and biased opinions), particularly compared with medical literature data. Anyone can post on social media, and so the derived content may be from individuals who may have other implant-specific issues such as capsular contracture or implant infection. Thus, understanding the diseases, disorders, symptoms, signs, etc, associated with a drug, disease, or medical procedure from social media data would always be at risk from confounders or errors. However, given that the medical knowledge and literature on BII have not been well established, and the related concepts are not well defined or well accepted, using social media data to understand emerging issues could be a meaningful starting point. Still, any findings from social media data would require a rigorous evaluation and validation based on medical and biological knowledge, experiments, clinical practice, etc. In addition, we have only analyzed 3, though the most relevant and prolific websites dedicated to BII discussions. A more comprehensive analysis of social media data on a much larger scale would be beneficial to better understand BII in a larger, diverse population. Sentiment analysis of social media data could be another valuable analysis to enable more insights into the health experiences of users or patients and their emotions or feelings. We will consider sentiment analysis in our future research when BII is better understood, and we can accurately annotate social media data.

\section{Conclusions}

This study has important implications for future methodological and clinical research. Future methodological research on NLP could include causality inference between BII and symptom and sign mentions from social media to understand their relations, etc. Our findings could provide the relevant domains for clinical research studies seeking to develop measures of BII and to identify its causes. More specifically, our results can provide a patient-derived definition of BII, which can be useful to clinicians treating patients with BII concerns to use this patient-centered language. Our methods and informatics strategies applied in this study would also provide working examples for analyzing other emerging but not well-defined illnesses from social media data.

Our analysis of social media data identified mentions such as rupture, infection, inflammation, pain, and fatigue, which were common self-reported issues on social media sites dedicated to BII. In addition, our analysis showed that a significant number of user comments and posts were also concerned with mental and physical health and toxicity issues after having breast implants. The findings from our study could be used to further the scientific study of BII, as well as the care of patients presenting with the described symptoms, by allowing clinicians to develop a patient-centered language to better approach the patients with concerns. Our study provides the first analysis and derived knowledge of BII from social media using NLP techniques and demonstrates the potential of using social media information to better understand emerging illnesses.

\section{Acknowledgments}

$\mathrm{XN}$ conceived the research, obtained funding for the research, and supervised VD; PK, MN, and CL provided substantial medical background and insights; Vishal Dey and Xia Ning conducted the research, including data curation, methodology design and implementation, and analysis; Vishal Dey drafted the original manuscript; Vishal Dey and Xia Ning conducted the manuscript editing; PK, MN, and CL reviewed the manuscript and provided constructive suggestions and feedback.

\section{Conflicts of Interest}

None declared.

\section{Multimedia Appendix 1}

A brief description of latent Dirichlet allocation. 


\section{References}

1. Barros JM, Duggan J, Rebholz-Schuhmann D. The application of internet-based sources for public health surveillance (Infoveillance): systematic review. J Med Internet Res 2020 Mar 13;22(3):e13680 [FREE Full text] [doi: 10.2196/13680] [Medline: 32167477]

2. Schillinger D, Chittamuru D, Ramírez AS. From "Infodemics" to health promotion: a novel framework for the role of social media in public health. Am J Public Health 2020 Sep;110(9):1393-1396. [doi: 10.2105/AJPH.2020.305746] [Medline: 32552021]

3. Li D, Chaudhary H, Zhang Z. Modeling spatiotemporal pattern of depressive symptoms caused by COVID-19 using social media data mining. Int J Environ Res Public Health 2020 Jul 10;17(14):4988 [FREE Full text] [doi: 10.3390/ijerph17144988] [Medline: 32664388]

4. Shen C, Chen A, Luo C, Zhang J, Feng B, Liao W. Using reports of symptoms and diagnoses on social media to predict COVID-19 case counts in mainland china: observational infoveillance study. J Med Internet Res 2020 May 28;22(5):e19421 [FREE Full text] [doi: 10.2196/19421] [Medline: 32452804]

5. Aiello AE, Renson A, Zivich PN. Social media- and internet-based disease surveillance for public health. Annu Rev Public Health 2020 Apr 02;41:101-118. [doi: 10.1146/annurev-publhealth-040119-094402] [Medline: $\underline{31905322]}$

6. Li C, Chen LJ, Chen X, Zhang M, Pang CP, Chen H. Retrospective analysis of the possibility of predicting the COVID-19 outbreak from Internet searches and social media data, China, 2020. Euro Surveill 2020 Mar;25(10):2000199 [FREE Full text] [doi: 10.2807/1560-7917.ES.2020.25.10.2000199] [Medline: 32183935]

7. Ginsberg J, Mohebbi MH, Patel RS, Brammer L, Smolinski MS, Brilliant L. Detecting influenza epidemics using search engine query data. Nature 2009 Feb 19;457(7232):1012-1014. [doi: 10.1038/nature07634] [Medline: 19020500]

8. Naslund JA, Grande SW, Aschbrenner KA, Elwyn G. Naturally occurring peer support through social media: the experiences of individuals with severe mental illness using YouTube. PLoS One 2014;9(10):e110171 [FREE Full text] [doi: 10.1371/journal.pone.0110171] [Medline: 25333470]

9. Foufi V, Timakum T, Gaudet-Blavignac C, Lovis C, Song M. Mining of textual health information from reddit: analysis of chronic diseases with extracted entities and their relations. J Med Internet Res 2019 Jun 13;21(6):e12876 [FREE Full text] [doi: $\underline{10.2196 / 12876}$ ] [Medline: $\underline{31199327}$ ]

10. Wakamiya S, Morita M, Kano Y, Ohkuma T, Aramaki E. Tweet classification toward twitter-based disease surveillance: new data, methods, and evaluations. J Med Internet Res 2019 Feb 20;21(2):e12783. [doi: 10.2196/12783] [Medline: 30785407]

11. Attai DJ, Cowher MS, Al-Hamadani M, Schoger JM, Staley AC, Landercasper J. Twitter social media is an effective tool for breast cancer patient education and support: patient-reported outcomes by survey. J Med Internet Res 2015;17(7):e188 [FREE Full text] [doi: 10.2196/jmir.4721] [Medline: 26228234]

12. Osadchiy V, Mills JN, Eleswarapu SV. Understanding patient anxieties in the social media era: qualitative analysis and natural language processing of an online male infertility community. J Med Internet Res 2020 Mar 10;22(3):e16728 [FREE Full text] [doi: 10.2196/16728] [Medline: $\underline{32154785}$ ]

13. Nobles AL, Leas EC, Althouse BM, Dredze M, Longhurst CA, Smith DM, et al. Requests for diagnoses of sexually transmitted diseases on a social media platform. J Am Med Assoc 2019 Nov 05;322(17):1712-1713. [doi: 10.1001/jama.2019.14390] [Medline: 31688875]

14. Kahlor L, Mackert M. Perceptions of infertility information and support sources among female patients who access the internet. Fertil Steril 2009 Jan;91(1):83-90. [doi: 10.1016/j.fertnstert.2007.11.005] [Medline: 18243181]

15. Guntuku SC, Yaden DB, Kern ML, Ungar LH, Eichstaedt JC. Detecting depression and mental illness on social media: an integrative review. Curr Opin Behav Sci 2017 Dec;18:43-49. [doi: 10.1016/j.cobeha.2017.07.005]

16. Karmen C, Hsiung RC, Wetter T. Screening internet forum participants for depression symptoms by assembling and enhancing multiple NLP methods. Comput Methods Programs Biomed 2015 Jun;120(1):27-36. [doi: 10.1016/j.cmpb.2015.03.008] [Medline: 25891366]

17. Dreisbach C, Koleck TA, Bourne PE, Bakken S. A systematic review of natural language processing and text mining of symptoms from electronic patient-authored text data. Int J Med Inform 2019 May;125:37-46 [FREE Full text] [doi: 10.1016/j.ijmedinf.2019.02.008] [Medline: 30914179]

18. Chapman B, Raymond B, Powell D. Potential of social media as a tool to combat foodborne illness. Perspect Public Health 2014 Jul;134(4):225-230. [doi: 10.1177/1757913914538015] [Medline: 24990140]

19. Harris JK, Mansour R, Choucair B, Olson J, Nissen C, Bhatt J, Centers for Disease ControlPrevention. Health department use of social media to identify foodborne illness - Chicago, Illinois, 2013-2014. MMWR Morb Mortal Wkly Rep 2014 Aug 15;63(32):681-685 [FREE Full text] [Medline: 25121710]

20. Casas J, Mugellini E, Abou K. Early detection of foodborne illnesses in social media. In: Proceedings of the 2nd International Conference on Human Interaction and Emerging Technologies: Future Applications (IHIET-AI 2020). Lausanne, Switzerland: Springer; 2020 Presented at: 2nd International Conference on Human Interaction and Emerging Technologies: Future Applications (IHIET-AI 2020); April 23-25, 2020; Lausanne, Switzerland p. 415-420. [doi: 10.1007/978-3-030-44267-5 62] 
21. McGough SF, Brownstein JS, Hawkins JB, Santillana M. Forecasting zika incidence in the 2016 Latin America outbreak combining traditional disease surveillance with search, social media, and news report data. PLoS Negl Trop Dis 2017 Jan;11(1):e0005295 [FREE Full text] [doi: 10.1371/journal.pntd.0005295] [Medline: 28085877]

22. Zhao J, Han H, Zhong B, Xie W, Chen Y, Zhi M. Health information on social media helps mitigate Crohn's disease symptoms and improves patients' clinical course. Comput Hum Behav 2021 Feb;115:106588. [doi: 10.1016/j.chb.2020.106588]

23. Pandrekar S, Chen X, Gopalkrishna G, Srivastava A, Saltz M, Saltz J, et al. Social media based analysis of opioid epidemic using Reddit. In: AMIA Annu Symp Proc. 2018 Presented at: AMIA Annual Symposium; November 3-7, 2018; San Francisco, CA p. 867-876 URL: http://europepmc.org/abstract/MED/30815129

24. Marques-Toledo CD, Degener CM, Vinhal L, Coelho G, Meira W, Codeço CT, et al. Dengue prediction by the web: tweets are a useful tool for estimating and forecasting Dengue at country and city level. PLoS Negl Trop Dis 2017 Jul;11(7):e0005729 [FREE Full text] [doi: 10.1371/journal.pntd.0005729] [Medline: 28719659]

25. Signorini A, Segre AM, Polgreen PM. The use of Twitter to track levels of disease activity and public concern in the U.S. during the influenza A H1N1 pandemic. PLoS One 2011;6(5):e19467 [FREE Full text] [doi: 10.1371/journal.pone.0019467] [Medline: 21573238]

26. Broniatowski DA, Paul MJ, Dredze M. National and local influenza surveillance through Twitter: an analysis of the 2012-2013 influenza epidemic. PLoS One 2013;8(12):e83672 [FREE Full text] [doi: 10.1371/journal.pone.0083672] [Medline: 24349542]

27. Klembczyk JJ, Jalalpour M, Levin S, Washington RE, Pines JM, Rothman RE, et al. Google flu trends spatial variability validated against emergency department influenza-related visits. J Med Internet Res 2016;18(6):e175 [FREE Full text] [doi: 10.2196/jmir.5585] [Medline: 27354313]

28. Eysenbach G. Infodemiology and infoveillance: framework for an emerging set of public health informatics methods to analyze search, communication and publication behavior on the internet. J Med Internet Res 2009;11(1):e11 [FREE Full text] [doi: 10.2196/jmir.1157] [Medline: 19329408]

29. Li J, Xu Q, Cuomo R, Purushothaman V, Mackey T. Data mining and content analysis of Chinese social media platform Weibo during early COVID-19 outbreak: a retrospective observational infoveillance study. JMIR Public Health Surveill 2020 Apr 14;6(2):e18700 [FREE Full text] [doi: 10.2196/18700] [Medline: 32293582]

30. Huang C, Xu X, Cai Y, Ge Q, Zeng G, Li X, et al. Mining the characteristics of COVID-19 patients in China: analysis of social media posts. J Med Internet Res 2020 May 17;22(5):e19087 [FREE Full text] [doi: 10.2196/19087] [Medline: $\underline{32401210]}$

31. 2019 Plastic Surgery Statistics Report. American Society of Plastic Surgeons (ASPS). 2019. URL: https://www. plasticsurgery.org/news/plastic-surgery-statistics?sub=2019+Plastic+Surgery+Statistics [accessed 2021-03-29]

32. 2018 National Plastic Surgery Statistics. American Society of Plastic Surgeons (ASPS). 2018. URL: https://www. plasticsurgery.org/documents/News/Statistics/2018/plastic-surgery-statistics-report-2018.pdf [accessed 2019-07-18]

33. Balk EM, Earley A, Avendano EA, Raman G. Long-term health outcomes in women with silicone gel breast implants: a systematic review. Ann Intern Med 2016 Feb 02;164(3):164-175. [doi: 10.7326/M15-1169] [Medline: 26550776]

34. Watad A, Rosenberg V, Tiosano S, Tervaert JW, Yavne Y, Shoenfeld Y, et al. Silicone breast implants and the risk of autoimmune/rheumatic disorders: a real-world analysis. Int J Epidemiol 2018 Dec 01;47(6):1846-1854. [doi: 10.1093/ije/dyy217] [Medline: 30329056]

35. Labadie JG, Korta DZ, Barton N, Mesinkovska NA. Cutaneous hypersensitivity-like reactions associated with breast implants: a review. Dermatol Surg 2018 Mar;44(3):323-329. [doi: 10.1097/DSS.0000000000001448] [Medline: 29293108]

36. Calobrace MB, Stevens WG, Capizzi PJ, Cohen R, Godinez T, Beckstrand M. Risk factor analysis for capsular contracture: a 10-year sientra study using round, smooth, and textured implants for breast augmentation. Plast Reconstr Surg 2018 Apr;141(4S):20-28. [doi: 10.1097/PRS.0000000000004351] [Medline: 29595715]

37. Rohrich RJ, Kaplan J, Dayan E. Silicone implant illness: science versus myth? Plast Reconstr Surg 2019;144(1):98-109. [doi: 10.1097/prs.0000000000005710]

38. Coroneos C, Selber J, Offodile A, Butler C, Clemens M. US FDA breast implant postapproval studies: long-term outcomes in 99,993 patients. Ann Surg 2019 Jan;269(1):30-36. [doi: 10.1097/SLA.0000000000002990] [Medline: 30222598]

39. Gabriel SE, O'Fallon WM, Kurland LT, Beard CM, Woods JE, Melton LJ. Risk of connective-tissue diseases and other disorders after breast implantation. N Engl J Med 1994 Jun 16;330(24):1697-1702. [doi: 10.1056/NEJM199406163302401] [Medline: $\underline{8190133]}$

40. Peters W, Smith D, Fornasier V, Lugowski S, Ibanez D. An outcome analysis of 100 women after explantation of silicone gel breast implants. Ann Plast Surg 1997 Jul;39(1):9-19. [doi: 10.1097/00000637-199707000-00002] [Medline: 9229086]

41. Janowsky EC, Kupper LL, Hulka BS. Meta-analyses of the relation between silicone breast implants and the risk of connective-tissue diseases. N Engl J Med 2000 Mar 16;342(11):781-790. [doi: 10.1056/NEJM200003163421105] [Medline: $\underline{10717013}$

42. Rohrich RJ, Kenkel JM, Adams WP, Beran S, Conner WC. A prospective analysis of patients undergoing silicone breast implant explantation. Plast Reconstr Surg 2000 Jun;105(7):2529-2538. [doi: 10.1097/00006534-200006000-00036] [Medline: $\underline{10845310]}$ 
43. Nahabedian MY, Tsangaris T, Momen B, Manson PN. Infectious complications following breast reconstruction with expanders and implants. Plast Reconstr Surg 2003 Aug;112(2):467-476. [doi: 10.1097/01.PRS.0000070727.02992.54] [Medline: 12900604$]$

44. Siggelkow W, Klosterhalfen B, Klinge U, Rath W, Faridi A. Analysis of local complications following explantation of silicone breast implants. Breast 2004 Apr;13(2):122-128. [doi: 10.1016/j.breast.2003.08.003] [Medline: 15019692]

45. Lee I, Cook NR, Shadick NA, Pereira E, Buring JE. Prospective cohort study of breast implants and the risk of connective-tissue diseases. Int J Epidemiol 2011 Feb;40(1):230-238 [FREE Full text] [doi: 10.1093/ije/dyq164] [Medline: 20943932]

46. Tang SY, Israel JS, Afifi AM. Breast implant illness: symptoms, patient concerns, and the power of social media. Plast Reconstr Surg 2017 Nov;140(5):765-766. [doi: 10.1097/PRS.0000000000003785] [Medline: 28753149]

47. Tang SY, Israel JS, Poore SO, Afifi AM. Facebook facts: breast reconstruction patient-reported outcomes using social media. Plast Reconstr Surg 2018 May;141(5):1106-1113. [doi: 10.1097/PRS.0000000000004275] [Medline: 29697604]

48. Magnusson MR, Cooter RD, Rakhorst H, McGuire PA, Adams WP, Deva AK. Breast implant illness: a way forward. Plast Reconstr Surg 2019 Mar;143(3S):74-81. [doi: 10.1097/PRS.0000000000005573] [Medline: 30817559]

49. Adidharma W, Latack KR, Colohan SM, Morrison SD, Cederna PS. Breast implant illness: are social media and the internet worrying patients sick? Plast Reconstr Surg 2020 Jan;145(1):225-227. [doi: 10.1097/PRS.0000000000006361] [Medline: $\underline{31625990]}$

50. Keane G, Chi D, Ha A, Myckatyn T. En bloc capsulectomy for breast implant illness: a social media phenomenon? Aesth Surg J 2021;41(4):448-459. [doi: 10.1093/asj/sjaa203]

51. Wee CE, Younis J, Isbester K, Smith A, Wangler B, Sarode AL, et al. Understanding breast implant illness, before and after explantation: a patient-reported outcomes study. Ann Plast Surg 2020 Jul;85(S1 Suppl 1):82-86 [FREE Full text] [doi: 10.1097/SAP.0000000000002446] [Medline: 32530850]

52. Lee M, Ponraja G, McLeod K, Chong S. Breast implant illness: a biofilm hypothesis. Plast Reconstr Surg Glob Open 2020 Apr;8(4):e2755. [doi: 10.1097/GOX.0000000000002755] [Medline: 32440423]

53. Blog - Cancer.net. URL: https://www.cancer.net/blog [accessed 2021-01-05]

54. Blog - Living Beyond Breast Cancer. URL: https://www.lbbc.org/blog [accessed 2021-01-05]

55. Stories from patients with breast, lung and other cancers. Cancer Treatment Centers of America. URL: https://www. cancercenter.com/patient-stories [accessed 2021-01-05]

56. Chronic illness. Mighty Well Archives. URL: https://blog.mighty-well.com/category/chronic-illness/ [accessed 2021-01-05]

57. Practical pain management - symptoms, causes, treatments, medications for chronic pain. Remedy Health Media, LLC. URL: https://www.practicalpainmanagement.com/patients [accessed 2021-01-05]

58. Resources: for patients. Body Politic. URL: https://www.wearebodypolitic.com/resources [accessed 2021-01-05]

59. Coronavirus blog team. Medium. URL: https://medium.com/@ coronavirus blog team [accessed 2021-01-05]

60. COVID-19 patient stories. Johns Hopkins Medicine. URL: https://www.hopkinsmedicine.org/coronavirus/patient-stories/ [accessed 2021-01-05]

61. About breast implant illness. Breast Implant Illness. URL: https://www.breastimplantillness.com/symptoms/ [accessed 2019-05-10]

62. Breast implant illness - symptoms, explant, surgeons, detox. Healing Breast Implant Illness. URL: https:/ /healingbreastimplantillness.com [accessed 2019-05-10]

63. \#breastimplantillness hashtag on Instagram. Instagram. URL: https://www.instagram.com/explore/tags/breastimplantillness [accessed 2019-09-05]

64. Savova GK, Masanz JJ, Ogren PV, Zheng J, Sohn S, Kipper-Schuler KC, et al. Mayo clinical Text Analysis and Knowledge Extraction System (cTAKES): architecture, component evaluation and applications. J Am Med Inform Assoc 2010;17(5):507-513 [FREE Full text] [doi: 10.1136/jamia.2009.001560] [Medline: 20819853]

65. Bodenreider O. The Unified Medical Language System (UMLS): integrating biomedical terminology. Nucleic Acids Res 2004 Jan 1;32(Database issue):267-270 [FREE Full text] [doi: 10.1093/nar/gkh061] [Medline: 14681409]

66. Blei DM, Ng AY, Jordan MI. Latent Dirichlet allocation. J Mach Learn Res 2003;3:993-1022. [doi: 10.1016/B978-0-12-411519-4.00006-9]

67. Loper E, Bird S. NLTK: the Natural Language Toolkit. In: Proceedings of the ACL-02 Workshop on Effective Tools and Methodologies for Teaching Natural Language Processing and Computational Linguistics. USA: Association for Computational Linguistics; 2002 Presented at: ACL-02 Workshop on Effective Tools and Methodologies for Teaching Natural Language Processing and Computational Linguistics; July 7, 2002; Philadelphia Pennsylvania p. 63-70. [doi: $\underline{10.3115 / 1118108.1118117]}$

68. Blei DM. C implementation of variational EM for latent Dirichlet Allocation (LDA). Github. 2013. URL: https://github. com/blei-lab/lda-c [accessed 2019-07-02]

69. Hofmann T. Probabilistic latent semantic indexing. In: Proceedings of the 22nd Annual International Acm Sigir Conference on Research and Development in Information Retrieval. United States: Association for Computing Machinery; 1999 Presented at: SIGIR99: 22nd Annual International ACM SIGIR Conference on Research and Development in Information Retrieval; August 15 - 19, 1999; Berkeley California USA p. 50-57. [doi: 10.1145/312624.312649] 
70. Blei D, Lafferty J. Correlated topic models. In: Proceedings of the Advances in Neural Information Processing Systems. Cambridge, United States: MIT Press; 2006 Presented at: Advances in Neural Information Processing Systems; December 4-7, 2006; Vancouver, Canada. [doi: 10.5555/2976248.2976267]

71. Chang J, Boyd-Graber J, Gerrish S, Wang C, Blei D. Reading tea leaves: how humans interpret topic models. In: Proceedings of the Advances in Neural Information Processing Systems. United States: Curran Associates Inc; 2009 Presented at: Advances in Neural Information Processing Systems; December 2009; Vancouver, Canada. [doi: 10.5555/2984093.2984126]

\section{Abbreviations}

ASCII: American Standard Code for Information Interchange

BII: breast implant illness

cTAKES: clinical Text Analysis and Knowledge Extraction System

CUI: concept unique identifier

LDA: latent Dirichlet allocation

NLP: natural language processing

UMLS: unified medical language system

XMI: XML metadata interchange

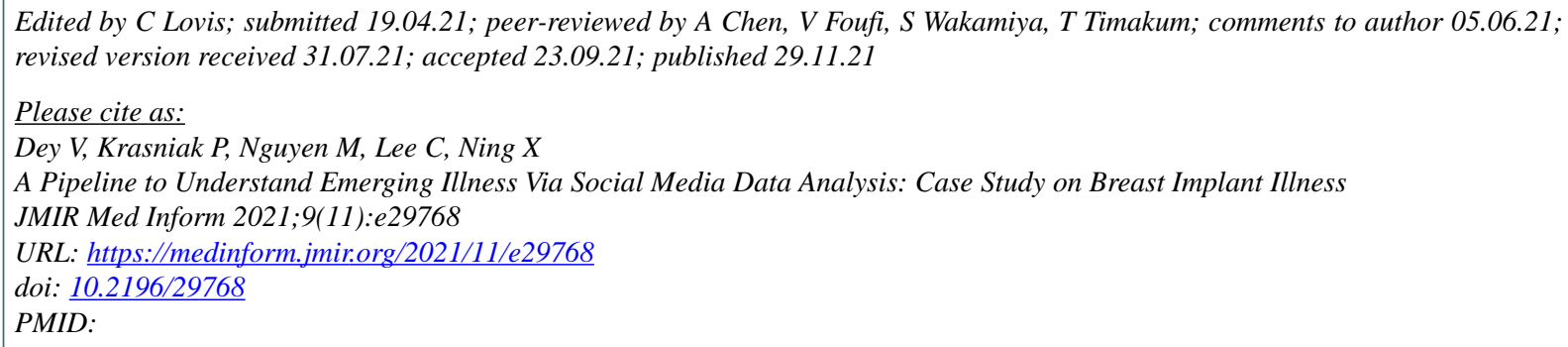

(C) Vishal Dey, Peter Krasniak, Minh Nguyen, Clara Lee, Xia Ning. Originally published in JMIR Medical Informatics (https://medinform.jmir.org), 29.11.2021. This is an open-access article distributed under the terms of the Creative Commons Attribution License (https://creativecommons.org/licenses/by/4.0/), which permits unrestricted use, distribution, and reproduction in any medium, provided the original work, first published in JMIR Medical Informatics, is properly cited. The complete bibliographic information, a link to the original publication on https://medinform.jmir.org/, as well as this copyright and license information must be included. 